Available online on 15.06.2020 at http://ajprd.com
(C) 2013-20, publisher and licensee AJPRD, This is an Open Access article which permits unrestricted non-
commercial use, provided the original work is properly cited

Open $\overbrace{\text { Access }}$

Review Article

\title{
Emulgel: A Topical Preparation
}

\section{Nikhita Parihar*, Mahendra Saini, Shankar Lal Soni, Vandana Sharma.}

Arya College of Pharmacy, Kukas, Jaipur, Rajasthan - 302028, India.

\section{A B S T R A C T}

\begin{abstract}
Emulgel is an emerging topical drug formulation which is becoming increasingly popular due to its advantages over the conventional topical preparations. The emulgel is a combination of an emulsion and a gel and thus has a dual release control system. Its biggest and most favorable advantage has been the ability to incorporate hydrophobic drugs, thus making it emerge as a more popular choice these days. The emulgel is also greaseless, transparent; it can be easily spread and removed, has a long shelf- life, is thixotropic and is also pleasant looking. The emulgel is turning out be a preferred choice for cosmetic and dermatological preparations and its use and application will accelerate in the coming future.
\end{abstract}

KEYWORDS: Emulgels, hydrophobic drugs, topical drug delivery.

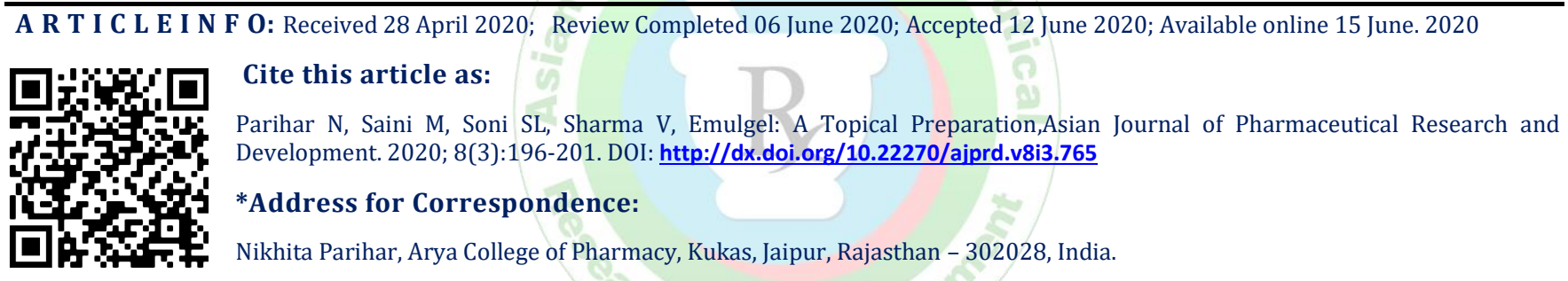

\section{INTRODUCTION}

A topical drug is delivered or applied on the surface of the human body such as the skin or the mucous membrane via a vast extent of classes including creams, foams, gels, lotions, and ointments. ${ }^{1}$ They are applied on a very large scale spectrum of both cosmetic and dermatological applications that can be for healthy as well as diseased skin. ${ }^{2}$ Drug substances are rarely administered alone, but somewhat as a part of a formulation, in combination with one or more non medicated agents that serve different and specialized pharmaceutical function. Some topical administrations are applied on the skin but they actually work towards being absorbed systemically. ${ }^{3}$ Drug absorption through the skin is augmented if we have the drug substance in the solution form or if the lipid/water partition coefficient is favorable and the last criteria could be that it is a non- electrolyte. Antiseptics, antifungal agents, skin emollients and protectant are some of the type of drugs which are used for their localized actions. There are many advantages of tropical drug delivery systems such as avoidance of first pass metabolism and gastrointestinal incompatibility. Their major advantage being that they increase patient compliance and the patient can easily self- administer these medications without any dependence on another person. They also have short biological half- life and a slight therapeutic window and thus can easily be stopped when required. ${ }^{4,5}$

Human skin is a unique organ that allows the terrestrial life to regulate its heat and water loss from the body and at the same time it also prevents the entry of harmful chemicals or microorganisms. The skin is the largest organ of the human body and covers almost $10 \%$ of the total body surface and approximately covers an average area of $1.7 \mathrm{~m}^{2}$. Being such a large and easily accessible organ, the skin has many ideal as well as multiple sites to administer therapeutic agents for both local and systemic actions. The human skin is also a very highly efficient self- repairing barrier to the contaminants of the outside and it keeps the insides of the human body inside and separate from these external parameters. $^{6}$

Gels are semi- solid preparations which have varying properties such as being soft and weak to hard and tough. ${ }^{7}$ Gels are considerably dilute cross- linked system, which do 
not exhibit any flow when they are in a steady state. ${ }^{8}$ Gels are actually a dispersion of molecules of a liquid within a solid medium. Gels are also easily acceptable because of their cosmetic elegance. They are designed by entrapment of large quantities of aqueous or hydro alcoholic liquid in a network of colloidal solid particles, which may encompass of inorganic substances, such as aluminum salts or organic polymers of synthetic or natural origin. ${ }^{9}$ They possess a higher aqueous component that permits greater dissolution of drugs and thuseffortless migration of the drug through a vehicle which is actually a liquid, as compared to the ointment or cream bases. ${ }^{10}$ As gels have many advantages, their major disadvantage is in the delivery of hydrophobic drugs. Thus to prevail over this limitation the emulgels are formed so that they can easily deliver these hydrophobic drugs too. A classical emulsion is convertedinto an emulgel due to the presence of a gelling agent in the water phase. ${ }^{11}$ We use both water-in-oil emulsions and oil-in water emulsions as vehicles to deliver various types of drugs to the skin.

For dermatological use, the emulgels possess severaladvantages such as being greaseless, easily spreadable, transparent, easily removable, thixotropic, emollient, non-staining, bio-friendly,long shelf life and a pleasing appearance. ${ }^{2}$ Molecules can penetrate the skin mainly by three routes which include the intact stratum corneum, through the sweat ducts or through sebaceous follicles. For percutaneous drug absorption, the stratum corneum's surface presents more than $99 \%$ of the total skin surface for this purpose. ${ }^{12}$

There are many steps which are involved in percutaneous absorption which mainly include the establishment of a concentration gradient which is the main driving force for the drug movement across the skin, drug diffusion across the layers of the skin (diffusion coefficient) and release of drug from the vehicle (partition coefficient). The topical drugs need to have ideally low molecular mass (600Da), adequate solubility in oil and water, and a high partition coefficient. But there are a few molecules which do not penetrate the stratumcorneum such as very small particles, water soluble ions and polar molecules. These topical formulations can also be used as barriers for the skin such as sun screening agents which protect the skin from harmful ultraviolet radiation and many emollient preparations which restore the pliability to a desiccated horny layer. ${ }^{13}$

As we make these semi solid preparations for cutaneous application, we also need to add anti- microbial preservative in the formulation and the preservative selected should satisfy the need and efficacy in the product that is required by the competent authority. A suitable test method is also required to determine or judge the efficacy of the preservative for the formulation. The sterile semisolid preparations for cutaneous application are prepared using materials and methods that have been designed to make sure that sterility is established and that the introduction of contaminants and the growth of microorganisms is avoided. ${ }^{14}$ The effectiveness of the antimicrobial agent can be determined by various factors such as the active constituent of the preparation or by the formulation in which it is incorporated or based on the container and closure used. The preparations should always be checked for sterility. ${ }^{15}$ Theemulgels are thus being used for many skin disorders especially the fungal infections and many new emulgels are getting formulated to be used for acne in adolescents, thus sterility tests are a must for them. ${ }^{16}$

\section{RATIONALE FOR USING EMULGEL}

There are numerous medicated products available in the market that are applied to the skin or mucous membrane to enhance its quality or restore its fundamental function or pharmacologically alter an action in the underlined tissues etc. but these topical or dermatological products that are available mostly in the form of ointments, creams or lotions, have plenty of disadvantages. These products are sticky in nature and cause uneasiness to the patient when applied, they also have minimal spreading coefficient that is why they need to be rubbed when applied and these preparations also exhibit the problem of stability. They are also sometimes time consuming to apply and regimen may also become difficult when lots of formulations are prescribed. ${ }^{17}$ The use of transparent gels has increasingly expanded in the pharmaceutical and cosmetic preparations because there are lots of limitations within the major group of semisolid preparations. But in spite of many advantages of gels, their biggest limitation is the delivery of hydrophobic drugs. That is why to overcome this sole limitation an emulsion based approach is being developed and used so that even the hydrophobic drugs could be successfully incorporated and delivered through gels. ${ }^{18}$

\section{PHYSIOLOGY OF SKIN}

As most of the topical preparations including emulgels are meant to be applied on the surface of the skin, thus it is required to have a basic knowledge of the skin and its physiology function fordesigning the most appropriate and acceptable preparation. The average adult human body covers a surface area of approximately $2 \mathrm{~m}^{2}$ and therefore receives one - third of the blood circulating the human body. The skin also relatively forms a waterproof layer that protects the deeper and more delicate structures.There are approximately $40-70$ hair follicles and 200 to 300 sweat ducts on every square centimeter of the average human skin. The $\mathrm{pH}$ of the skin also ranges from 4 to 5.6. The $\mathrm{pH}$ of the skin surface is also affected by the sweat and fatty acids that are secreted from the sebum. The skin is considered to have four distinctly layers of tissues which are:

1 Non-viable epidermis2 Viable epidermis

3 Viable dermis4 Subcutaneous connective tissue ${ }^{19,20}$

\section{FACTORS AFFECTING TOPICAL ABSORPTION OF DRUGS ${ }^{21,22}$}

\section{Physiological factors}

1. Skin thickness.

2. Lipid content.

3. Density of hair follicles.

4. Density of sweat glands.

5. Skin $\mathrm{pH}$. 
6. Blood flow.

7. Hydration of skin.

8. Inflammation of skin.

\section{Physiochemical factors}

1. Partition coefficient.

2. Molecular weight (<400 Dalton).

3. Degree of ionization (only unionized drugs gets absorbed well)

4. Effect of vehicles.

Factors that need to be considered when choosing a topical preparation ${ }^{23,24}$

1. Effect of the vehicle e.g. the penetration of the active ingredient is enhanced by an occlusive vehicle and also improves efficacy. The vehicle may have a cooling, drying, emollient or protective action.

2. The type of preparation should be synonymous with the type of lesions. For example, greasy ointments should be avoided for an acute weepy dermatitis.

3. The type of preparation should be complimentary with the site for example gel or lotion should be used for hairy areas of the body.

4. Irritation or sensitization potential of the topical preparation. It is a common finding that ointments and w/o creams are less irritating, while gels cause a slight irritation.

\section{Method to Enhance Drug Penetration and Absorption ${ }^{25}$}

1. Chemical enhancement

2. Physical enhancement

3. Biochemical enhancement

4. Supersaturation enhancement

Advantages ${ }^{26,27}$

Hydrophobic drugs can be easily integrated into gels using $\mathrm{d} / \mathrm{o} / \mathrm{w}$ emulsions. As most of the hydrophobic drugs have solubility as their barrier which increases the problems during the release of the drug and thus they cannot be integrated directly into gel base and so need to be used in the form of emulgel.Therefore, emulgel helps in the integration of hydrophobic drugs into the oil phase and then these oily globules are dispersed in aqueous phase which results inan $\mathrm{o} / \mathrm{W}$ emulsion and this emulsion then can be mixed into the gel base. This proves to be better stabilized.

Better stability: Emulgels are more stable as compared to other transdermal preparations. For example powders are hygroscopic in nature, creams have a tendency to exhibitphase inversion or breaking and ointment undergo rancidity due to oily base.

Better loading capacity: the other novel approaches like niosomes and liposomes are of nano size and therefore because of their vesicular structures the end product may result in a leakage and in lesser entrapment efficiency. But gels have a better loading capacity because of their vast network.
Production viability and low preparation cost: As no specialized instruments are required for the production of emulgels and its preparation process is composed of simpler and shorter steps, thus the production of emulgel is viable. Moreover materials used are easily procured and cheaper. Hence, the production cost of emulgels is low.

No intensive sonication: During the sonication process, there are chances of drug degradation and leakage and vesicular molecules need intensive sonication which would thus lessen their quality. But this does not happen in the case of emulgels because they don't need to go through the sonication process.

Controlled release: There are many drugs which have a shorter $\mathrm{t} 1 / 2$ and thus to overcome this limit emulgels can be used and their effect of those drugs can be prolonged.

\section{FORMULATION OF EMULGEL}

Vehicle: The vehicle to be used in the emulgel preparation has the following properties

- Efficiently deposit the drug on to the skin with uniform distribution.

- Release the drug so that it can move freely to the site of action.

- Delver the drug to the desired target site.

- It should be able to sustain a therapeutic drug level in the target tissue for an adequate amount of time to get the desired pharmacological effect.

- Aptly formulated for the anatomic site that needs to be treated.

- Cosmetically appealing to the patient.

Since the efficiency of the epidermal barrier is considerably good, therefore the amount of topical drug that passes the stratum corneum is generally low. The characteristics of the vehicle and also the active agent itself, influences the rate and extent of absorption. ${ }^{28}$

Aqueous Material: This forms the aqueous phase of the emulsion that will be used for the emulgel preparation. The most commonly used agents as aqueous materials are water, alcohols etc.

Oils: These agents form the oily phase of the emulsion that will be used for the emulgel preparation. For the emulsions that are externally applied, mineral oils are used; either alone or with soft or hard paraffin and they are extensively used as the vehicle for the drug and for their occlusive and sensory characteristics. ${ }^{29}$

Emulsifiers:Emulsifying agents such as Polyethylene glycol 40 stearate $^{30}$, Sorbitan mono- oleate (Span 80) ${ }^{31}$, Polyoxyethylenesorbitan mono- oleate (Tween 80) ${ }^{32}$, Stearic acid, Sodium stearate, etc. are used to encourage emulsification at the time of manufacturing the preparation and also to control the stability of the product so as to maintain its shelf life that could be days or months or years for commercial preparations. ${ }^{31}$

Gelling Agent: These are the agents which are used to increase the consistency of any dosage forms as well as used as thickening agent. ${ }^{33,} 34$ Some examples of gelling agents are Carbopol- 934, Carbopol- 940, HPMC- 2910, etc. 
Penetration Enhancers: These are the agents that partition into and interact with the constituents of the skin and induce a temporary and reversible increase in skin permeability. ${ }^{35}$ Some examples of penetration enhancers are oleic acid, lecithine, linoleic acid, clove oil, menthol, etc. ${ }^{36}$

\section{PREPARATION OF EMULGEL ${ }^{37}$}

STEP 1: Formulation of Emulsion either O/W or W/O type.

STEP 2: Formulation of gel base

STEP 3: Emulsion is incorporated into gel base with continuous stirring.

\section{CHARACTERIZATION OF EMULGELS}

\section{Physical Examination:}

The prepared emulgel formulations are to be perceived visually based on their color, thiehomogeneity, consistency and phase separation. ${ }^{38}$

\section{Spreadibility:}

The spreadibilityis determined by an apparatus that was suggested by Mutimer et al in the year 1956. This apparatus is modified according to our own requirements in the laboratory and then used for the study. The apparatus is made up of a wooden block, which has a pulley attached to it at one end.Now to determine the spreadibility by this method we base it upon the 'Slip' and 'Drag' characteristics of the sample emulgels. A ground glass slide is fixed on this wooden block and an emulgel sample of sbout $2 \mathrm{gm}$ is taken and placed on this ground slide. Then the emulgel sample is sandwiched between two glass slides, the one which has the sample and the other glass slide with the same dimensions as the above slide and this second slide also has a hook attached to it. A $1 \mathrm{Kg}$ weight is placed on the top of the two slides for 5 minutes to expel air and to provide a uniform film of the emulgel between the slides. If there is an excess of the emulgel, then it needs to be removed or scraped off carefully. The top plate is then subjected to a pull of $80 \mathrm{gm}$ with the help of a string that is attached to the hook and then the time is calculated in seconds for the top slide to cover a distance of $7.5 \mathrm{~cm}$ and that time is noted. A shorter interval indicates better spreadibility. ${ }^{39}$ Formula used for calculating spreadibility is:

\section{$\mathrm{S}=\mathrm{M} . \mathrm{L} / \mathrm{T}$}

Where, $\mathrm{S}=$ spreadability,

$\mathrm{M}=$ Weight tied to upper slide,

$\mathrm{L}=$ Length of glass slides

$\mathrm{T}=$ Time taken to separate the slides completely from each other.

\section{Extrudability study:}

This is also called as Tube Test. This empirical test is done to measure the force that is required to extrude the material out of the tube. In this the method applied for determination of applied shear in the region of the rheogram corresponds to a shear rate that is exceeding the yield value and also exhibiting consequent plug flow. In the present study that we are conducting, the method that we have selected for evaluating theemulgel formulation for extrudability is based upon the quantity of emulgel in percentage and the amount of emulgelthat is extruded from lacquered aluminum collapsible tube on application of a certain amount of weight in grams.This method is required to extrude at least $0.5 \mathrm{~cm}$ ribbon of emulgel in 10 seconds from the collapsible tube. More quantity extruded, better is extrudability. The measurements are taken thrice from theextrudability of each formulation and the average values are then determined. After obtaining the values the extrudability is calculated by using the following formula:

Extrudability $=$ Applied weight to extrude emulgel from tube (in gm) / Area (in $\mathrm{cm}^{2}$ )

\section{Globule size and its determination in emulgel:}

This is determined by Malvern zetasizer. We take $1.0 \mathrm{gm}$ of the sample and dissolve it in purified water and then itis agitated to get a homogeneous dispersion. Sample is injected to photocell of zetasizer. Mean globule diameter and distribution is then obtained. ${ }^{40}$

\section{Rheological studies:}

To carry out the rheological studies, we use a cone and plate viscometer with spindle 52 by Brookfield Engineering Laboratories. The various emulgel formulations are taken and their viscosity is determined at $25^{\circ} \mathrm{C}$ by using the above mentioned apparatus connected to a circulating water bath which is controlled thermostatically. ${ }^{41}$

\section{Swelling Index:}

For the determination of the swelling index of a prepared topical emulgel, a porous aluminum foil is taken and on it 1 $\mathrm{gm}$ of gel is placed and then it is placed separately in a 50 $\mathrm{ml}$ beaker containing $10 \mathrm{ml} 0.1 \mathrm{~N} \mathrm{NaoH}$. Then samples arewithdrawn from the beakers at different time intervals and then placed on a dru place for some time and then it's reweighed. Swelling index is calculated as follows:

Swelling Index $(\mathrm{SW}) \%=[(\mathrm{Wt}-\mathrm{Wo}) / \mathrm{Wo}] \times 100$. Where (SW) \% stands for Equilibrium percent swelling, Wt is weight of swollen emulgel after time $t$ and Wo is original weight of emulgel at zero time. ${ }^{42}$

\section{Ex- vivo Bio adhesive strength measurement of topical anemulgel:}

This process can also be termed as mice shaven skin since we precisely use it in this experiment.We measure the bioadhesive strength with the help of the modified method.Firstly we have to cut the fresh skin into pieces and then wash it with $0.1 \mathrm{~N}$ NaoHsolution. Then two glass slides are taken and two pieces of the skin are tied to them separately. Amongst those two slides, one is fixed on the wooden piece and the other is tied with the balance on right hand side. Then we have to add extra weight on the left hand pan so as to balance both the right as well as the left pans. Now we have to place the $1 \mathrm{gm}$ of topical emulgel sample between these two slides which contain the hairless pieces of skin and then the extra weight that is attached to the left pan is removed so that the two pieces of skin sandwich together and some extra pressure is applied to eliminate the presence of air. This balance needs to be kept as such in the same position for 5 minutes. After the above steps, we have to apply a certain amount of weight to the left hand pan and this need to be done slowly at 
$200 \mathrm{mg} / \mathrm{min}$, until the patch gets detached from the skin surface. The measure of bioadhesive strength of the emulgel is determined by the weight (gram force) required to detach this sample from the skin surface. The bioadhesive strength is calculated by using following formula. ${ }^{43}$

Bioadhesive Strength $=$ Weight required (in gram) $/$ Area $\left(\mathrm{cm}^{2}\right)$

\section{Drug content determination:}

Drug concentration in emulgelis measured by spectrophotometer. Drug content of emulgel is measuredby dissolving known quantity in solvent (methanol) by Sonication. Absorbance was measured after suitable dilution in UV/VIS spectrophotometer (UV-1700 CE, Shimadzu Corporation, Japan). ${ }^{43}$

\section{In Vitro release study:}

Franz diffusion cell is used for the drug release studies. Emulgel is applied onto the surface of egg membrane evenly. The egg membrane is secured between the donor and the receptor chamber of the diffusion cell. Then the receptor chamber is filled with freshly prepared PBS $(\mathrm{pH}$ 5.5) solution to solubilize the drug. The receptor chamber is stirred by a magnetic stirrer. The samples of the emulgels of $1.0 \mathrm{ml}$ aliquots need to be collected at suitable time intervals and a UV visible spectrophotometer is then used to analyze the appropriately diluted samples for drug content. To obtain the total amount of drug release at each time interval, cumulative corrections are done. To determine the cumulative amount of drug that is released across the egg membrane, time is used as a function. ${ }^{44}$

\section{Skin Irritation Test (Patch Test):}

Apply the preparation of emugel on the properly shaven skin of rat and adverse effects like change in color, skin morphology changes etc. are checked upto 24 hours. The total set of 8 rats can be used for the study. If no irritation takes place the test is passed. If the skin irritation symptom transpires in more than 2 rats, the study needs to be repeated.

\section{Stability Studies:}

Stability studies are performed based on the International Council of Harmonization guidelines. The formulations are stored in hot air oven at $37 \pm 2^{\circ}, 45 \pm 2^{\circ}$ and $60 \pm 2^{\circ}$ for a period of 3 months and UV- Visible spectrophotometer is used to analyze the samples for drug content in every two weeks for the duration of the experiment. The stability study is carried out by measuring the change in $\mathrm{pH}$ of gel at regular interval of time. ${ }^{45}$

\section{CONCLUSION}

In the forthcoming future years, the use of topical drug delivery systems will increase since they have a better acceptability among the patients. The emulgel possess an advantage compared to other formulations in terms of spreadibilty, adhesion, viscosity and extrusion and thus will become a popular choice in drug delivery systems. In addition to all the above advantages, they will become a solution for loading hydrophobic drugs in water soluble gel bases thus providing a suitable medium for them.

\section{REFERENCES}

1. Psoriasis-Treatment, Medical Reference, University of Maryland Medical System, 2009.

2. Kshirsagar N A, Drug Delivery Systems, Ind. J. Pharmacology. 2000; 32:S54- S61.

3. Rashmi M., 'Topical gel: A review', Pharmainfo.com, August vol. 2008.

4. Nayank SH, Nkhat PD, Yeole PG, the Indian Pharmacist.2004; 3(27):7-14

5. Devada P, Jain A, Vyas N, Jain S, 'Development of antifungal emulsion based gel for topical fungal infection', Int J Pharm Res Dev.2011; 3(2):18-25.

6. McGrath JA, Eady R \& Pope FM, 'Chapter 3: Anatomy and Organization of human skin', p 3.1 3.15.

7. K. Khademhosseini and U. Demirci Gels Handbook: Fundamentals, Properties and Applications, World Scientific Pub Co Inc, 2016.

8. Ferry, John D., 'Viscoelastic Properties of Polymers', New York: Wiley, 1980.

9. Kumar L, Verma R., 'In vitro evaluation of topical gel prepared using natural polymer', International Journal of Drug Delivery.2010; 2:5863.

10. Gennaro AR, ed. Remington: the Science and Practice of Pharmacy, Easton, Mack Publishing Company19th ed., 1995.

11. Rieger MM, Lachman L, Lieberman HA, Kanig JL, 'The Theory and Practice of Industrial Pharmacy', 3rd ed., PA Lea and Febiger, Philadelphia, pp.1986; 502-533.

12. Jain A, Deveda P, Vyas N, Chauhan J et al., 'Development of Antifungal Emulsion Based Gel for Topical Fungal Infection(S),' IJPRD. 2011; 2(12).

13. Bruton L, Keith P, Blumenthal D, Buxton Z., 'Goodman \& Gillman's Manual of Pharmacology and Therapeutics,' McGraw's Hill, pp. 2008; 1086-1094.

14. KoheiKyuki, Tomohisa Shibuya, Kaito Tsurumi Hajime Fujimura, 'AntiInflammatory effect of diclofenacsodium ointment (cream) in topical application', by Japan J. Phamacol. 1983; 33:121-132.

15. Principle of Skin Theraphy, from Dermweb.com.

16. P. Utreja, S. Jain, A.K. Tiwary, 'Localized delivery of paditaxel using elastic liposomes: formulation development and evaluation', Drug Delivery. 2011; 367-376.

17. Bolognia, Jorizzo et al., Dermatology edition, 1st Edition, Annemarie Uliasz, Mark Lebwohl. Chapter 129, other topical medications.

18. Cecv G., 'Preclinical characterisation of NSAIDs in ultradeformable carriers or conventional topical gels,' International journal of pharmaceutics, 2008.

19. Kanikkannan N, Kandimalla K, Lamba SS, Singh M, 'Structure activity relationship of chemical penetration enhancers in transdermal drug delivery,' Current med chem. 1999; 6:593-608.

20. Singh PB, Choudhary PK, 'Penetration enhancers for transfer drug delivery of systemic agents', J Pharm Res. 2007; 6:44-50.

21. Kalia YN, Guy RH, 'Modeling transdermal drug release', Adv. Drug Deliv. Rev. 2001; 48:159-72.

22. Ayub, CA, Gomes ADM, Lima MVC, et al, 'Topical Delivery of Fluconazole: In Vitro Skin Penetration and Permeation Using Emulsions as Dosage Forms', Drug. Dev. Ind. Pharm. 2007; 33:273280.

23. Gaur PK, Mishra S, Purohit S, Dave K., 'Transdermal Drug Delivery System: A Review', AJPCR. 2009; 2:14-20.

24. Subranayam N, Ghosal SK, Moulik SP, 'Enhanced In Vitro Percutaneous Absorption and In Vivo Anti-Inflammatory Effect of a Selective Cyclooxygenase Inhibitor Using Microemulsion', Drug Dev. and Industrial Pharm., 2005.

25. Pathan, I.B.; Setty, C.M., 'Chemical penetration enhancers for transdermal drug delivery systems', Trop J Pharm Res. 2009; 8:173179.

26. Rashmi, MS., 'Topical Gel: A Review', Pharmainfo.net, 2008.

27. Djordjevic J, Michniak B, Uhrich, Kathryn E, AAPS PharmSciTech. 2003; 5(4):1-12.

28. Bonacucina G, Cespi M, Palmieri GF, 'Characterization and Stability of Emulsion Gels Based on Acrylamide/Sodium AcryloyldimethylTaurate Copolymer', AAPS PharmSciTech. 2009; 10(2):34-45.

29. Curr AEB., 'Transdermal Drug Delivery: Penetration Enhancement Techniques', Heather. Drug Delivery. 2005; 5(2):23-33.

30. Curr AEB., 'Transdermal Drug Delivery: Penetration Enhancement Techniques', Heather. Drug Delivery. 2005; 2:23-33.

31. Rutrer N., 'Drug absorption through the skin: a mixed blessing', Arch Dis Child. 1987; 62:220-221. 
32. Zhang XL, Zhao R, Qian W., 'Preparation of an emulgel for treatment of aphthous ulcer on the basis of carbomers', Chinese Pharm. J. 1995; 30:417-418.

33. Mortazavi SA, Aboofazeli R., 'An Investigation into the Effect of Various Penetration Enhancers on Percutaneous Absorption of Piroxicam', Iranian Journal of Pharmaceutical Research. 2003; 135140.

34. Kumar, L.; Verma, R., Int. J Drug Delivery. 2010; 58-63.

35. Jacob SW, Francone CA., Structure and Function of Man, (2).

36. Swarbrick, J., Encyclopedia of pharmaceutical technology, 3rd ed., 1551.

37. Jain A, Gautam SP, Gupta, Jain S, 'Development and characterization of Ketoconazole emulgel for topical drug delivery', Der Pharmacia Sinica. 2010; 1(3):221- 231.

38. WB Saunders Co. Philadelphia. 1970; 55-60.

39. Gupta GD, Gound RS., 'Release rate of nimesulide from different gellants', Indian J Pharm Sci. 1999; 61(1):229-23.
40. Gondaliya DP and Pundarikakshudu K., Indian drugs.2002; 39:465473.

41. Sanjay, Jain BD, Padsalg A, Patel K, Mokale V, 'Formulation, development and evaluation of Fluconazole gel in various polymer bases', Asian J Pharm. 2007; 1:63-68.

42. Patel RP, Patel G, Baria A., 'Formulation and evaluation of transdermal patch of aceclofenac', International Journal Drug Del. 2009; 1(3):41- 51 .

43. Chaudhari P, Ajab A, Malpure P, Kolsure P, Sanap D, 'Development and in-vitro evaluation of thermo reversible nasal gel formulations of Rizatriptan benzoate', Indian J Pharm Edu. Res.2009; 43:55-62.

44. Masmoudi H, Piccerelle P, Le Dréau Y, Kister J., 'A rheological method to evaluate the physical stability of highly viscous pharmaceutical oil-in-water emulsions', Pharm Res.s 2006; 23(8):1937-47.

45. Tadros TF, 'Future developments in cosmetic formulations', International Journal Cosmetic Sciences. 1992; 14(3):93- 111. 